\title{
Recommendation of Attributes for Heart Disease Prediction using Correlation Measure
}

\author{
S.Chellammal, R. Sharmila
}

\begin{abstract}
Heart diseases are the major cause for human mortality rate. Correct diagnosis and treatment at an early stage will save people from heart disease and will decrease mortality rate due to heart problem. Since ten years various data mining techniques have been used to facilitate the prediction of heart diseases .In general prediction algorithms for trained with huge, known dataset to arrive at a classifier which then predicts the diseases for unknown data with the help of classifying attributes. These attributes also called as features. In this work relevant features are determined for heart disease prediction with known dataset using correlation measures. The results are presented.
\end{abstract}

Index Terms - Correlation method, relevant features, Prediction

\section{INTRODUCTION}

In health care industry, predicting heart disease is a challenging issue [1-3]. In early days medical tests such as Electrocardiogram (ECG) and blood tests have been used for predicting heart diseases. In addition to clinical tests, computer aided diagnosis systems, namely, patient information, medical diagnosis and medical images are being used for predicting heart diseases. Machine learning algorithms have significant role in predicting diseases [5]. Nowadays along with machine learning techniques, big data tools and technologies are being employed to handle unstructured data, huge size and speedy data [6]. In[7], we have proposed a conceptual framework for the prediction of diseases using parallel programming models in big data environment. It is pre-requisite to identify features that are relevant to the prediction of diseases. From, literature it is found that 13 attributes, namely, age, sex, cp, trestbps, chol, $f b s$, restecg, thalach, exang, oldpeak, slope, ca and thal are being used for predicting heart diseases. In this work, these features are analysed for their relevancy for prediction of heart disease using correlation technique. Prediction results obtained using three different classifiers namely Naive Bayes (NB) classifier, Multi Layer Perceptron (MLP) and Sequential Minimal Optimization (SMO) with the above attributes are presented. The results show that 10 attributes, namely, age, sex, cp, restecg, thalach, exang, oldpeak, slope, ca and thal are found as most relevant attributes in predicting heart diseases. Experiments have been conducted using the thus said classifiers with a typical data set obtained

Revised Manuscript Received on July 10, 2019

S.Chellammal, Bharathidasan University Constituent Arts and Science College, Navalurkuttapattu, Tiruchirappalli, Tamil Nadu, India. (E-mail: chelsganesh@gmail.com)

R. Sharmila, Bharathidasan University Constituent Arts and Science College, Navalurkuttapattu, Tiruchirappalli, Tamil Nadu, India. (E-mail: sharmiparam@gmail.com) from UCI repository. Accuracy obtained using different classifiers with different sets of attributes are reported in this paper. The paper is organized as follows. Section II gives research works that have their focus on feature selection for prediction of heart disease. Sections III describes the method, tool and dataset used for determining relevant features. Section IV presents results and discussion. Section $\mathrm{V}$ concludes the work.

\section{LITERATURE SURVEY}

In general, filter and wrapper methods [8] are being used for feature selection for predicting heart diseases. In filter methods where feature selection is independent of the prediction algorithm [10], different statistical factors such as Information Gain, Chi-square test, Fisher Score, Correlation, LDA (Linear Discriminant Analysis) and ANOVA (Analysis of Variance) are used for finding relevancy [9]. As wrapper methods are computationally very expensive, filter methods are frequently used in practice [11-12]. Hence we made an investigation on research works which employ filter methods.

From literature, several research works[13-20] have used the thirteen attributes, namely, age, sex, chest pain type ( $c p)$, resting blood pressure(trestbps), serum cholesterol(chol), fasting blood pressure(fbs), resting electrocardiographic results(restecg), maximum heart rate achieved( thalach), exercise induced angina(exang), ST depression induced by exercise relative to rest(oldpeak), the slope of the peak exercise ST segment(slope), number of major vessels colored by flourosopy(ca) and thalassemia(thal) for prediction of heart diseases. Also, various research works have found relevant features from these 13 attributes using filter techniques. For example in [21], the authors have used three attributes, namely, $c p, c a$ and thal for predicting heart diseases. In [22], six attributes namely $c p$, thalach, exang, oldpeak, ca and thal have been used for prediction. In [23], an alternate set of six attributes such as $c p$, restecg, ex, thalach, slope and thal have been used for prediction. In [24], seven attributes namely $c p$, restcg, exang, thalach, oldpeak, ca and that have been used for prediction. Also, in [25], an alternate set of seven attributes, namely, $c p$, restecg, exang, thalach, oldpeak, ca and thal have been used for prediction. In [26], eight attributes namely thalach, chol, $\boldsymbol{c a}$, exang, slope, oldpeak, restecg and sex have been used for prediction, In [4] nine attributes namely thal, ca, exang,

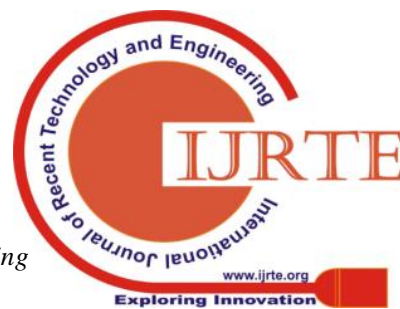




\section{RECOMMENDATION OF ATTRIBUTES FOR HEART DISEASE PREDICTION USING CORRELATION MEASURE}

thalach, oldpeak, cp, slope, sex and age have been used for prediction while in [27], eleven attributes, namely, age, $c p$, thal, trestbps, chol, ca, slope, restecg, fbs, oldpeak and ca used for prediction.
Table I highlights the relevant attributes used by different research works. Further for clarity, technique used for feature selection, data set, tool, classifier and its accuracy of prediction are provided in Table 1.

Table 1 Relevant attributes used by different research works

\begin{tabular}{|c|c|c|c|c|c|c|}
\hline \multirow[t]{2}{*}{ Ref. No } & \multirow[t]{2}{*}{$\begin{array}{l}\text { Attribute selection } \\
\text { method }\end{array}$} & \multirow[t]{2}{*}{ Selected attributes } & \multirow[t]{2}{*}{$\begin{array}{l}\text { No. of } \\
\text { attributes }\end{array}$} & \multirow[t]{2}{*}{$\begin{array}{l}\text { Dataset \& } \\
\text { tool }\end{array}$} & \multicolumn{2}{|c|}{$\begin{array}{l}\text { Classification accuracy } \\
\text { In } \%\end{array}$} \\
\hline & & & & & $\begin{array}{l}\text { With selected } \\
\text { attributes }\end{array}$ & With 13 attributes \\
\hline \multirow[t]{5}{*}{22} & \multirow{5}{*}{$\begin{array}{l}\text { CFS Subset } \\
\text { Evaluator } \\
\text { And three search } \\
\text { methods namely } \\
\text { Best First Search, } \\
\text { Rank search and } \\
\text { Genetic Search }\end{array}$} & \multirow{5}{*}{$\begin{array}{l}\text { chest pain type, max } \\
\text { heart rate, exercise } \\
\text { induced angina, } \\
\text { oldpeak, number of } \\
\text { major vessels } \\
\text { colored and thal }\end{array}$} & \multirow[t]{5}{*}{6} & \multirow{5}{*}{$\begin{array}{l}\text { Weka } 3.6 \\
\text { and } \\
\text { Cleveland } \\
\text { database } \\
\text { from UCI } \\
\text { repository. }\end{array}$} & $J 48-83.8284$ & $J 48-84.1584$ \\
\hline & & & & & $N B-84.1584$ & $N B-85.4785$ \\
\hline & & & & & $\begin{array}{l}\text { Logistic } \\
\text { Regression } \\
76.8977\end{array}$ & $\begin{array}{l}\text { Logistic Regression - } \\
77.2277\end{array}$ \\
\hline & & & & & $\begin{array}{l}\text { Classification } \\
\text { via regression - } \\
84.1584\end{array}$ & $\begin{array}{l}\text { Classification via } \\
\text { regression }-83.1683\end{array}$ \\
\hline & & & & & $S M O-84.4884$ & $S M O-82.8383$ \\
\hline 3 & $\begin{array}{ll}\text { CFS } & \text { Subset } \\
\text { Evaluator } & \end{array}$ & $\begin{array}{l}\text { gender, chest pain } \\
\text { type, cholesterol, } \\
\text { thal, exang and } \\
\text { oldpeak. }\end{array}$ & 6 & $\begin{array}{l}\text { Weka and } \\
\text { Hungarian } \\
\text { Heart } \\
\text { Disease } \\
\text { dataset } \\
\text { from UCI } \\
\text { repository }\end{array}$ & $S V M-89.4$ & SVM-97.9 \\
\hline 26 & $\begin{array}{l}\text { Binary Artificial } \\
\text { Bee Colony }\end{array}$ & $\begin{array}{l}\text { chest pain type, } \\
\text { resting blood } \\
\text { pressure, chol, max } \\
\text { heart rate achieved, } \\
\text { slope and thal }\end{array}$ & 6 & $\begin{array}{l}\text { Cleveland } \\
\text { data set } \\
\text { from UCI } \\
\text { repository. }\end{array}$ & $\begin{array}{l}B A B C-K N N- \\
92.4\end{array}$ & $\begin{array}{l}\text { (Please note: Results } \\
\text { are discussed only for } \\
6 \text { attributes) }\end{array}$ \\
\hline 21 & $\begin{array}{l}\text { CFS and Bayes } \\
\text { theorem }\end{array}$ & $\begin{array}{l}\text { chest pain type, } \\
\text { number of major } \\
\text { vessels colored and } \\
\text { thal }\end{array}$ & 3 & $\begin{array}{l}\text { Weka and } \\
\text { Statlog } \\
\text { dataset }\end{array}$ & $N B-85.18$ & $N B-83.70$ \\
\hline \multirow[t]{3}{*}{27} & \multirow{3}{*}{$\begin{array}{lr}\text { CFS } & \text { Subset } \\
\text { Evaluator } & \text { with } \\
\text { memory } & \text { based } \\
\text { classifier } & \end{array}$} & \multirow{3}{*}{$\begin{array}{l}\text { chest pain type, } \\
\text { resting } \\
\text { electrocardiographi } \\
\text { c results, exercised } \\
\text { induced angina, } \\
\text { maximum heart rate } \\
\text { achieved, oldpeak, } \\
\text { number of major } \\
\text { vessels colored and } \\
\text { thal }\end{array}$} & \multirow[t]{3}{*}{7} & \multirow[b]{3}{*}{$\begin{array}{l}\text { Open } \\
\text { source } \\
\text { machine } \\
\text { learning } \\
\text { tool and } \\
\text { Statlog } \\
\text { Heart } \\
\text { Disease } \\
\text { dataset UCI } \\
\text { from UCI } \\
\text { repository }\end{array}$} & $I B K-77.78$ & $I B K-74.75$ \\
\hline & & & & & K star -79.18 & K star -75.90 \\
\hline & & & & & $L W L-69.037$ & $L W L-71.10$ \\
\hline \multirow[t]{9}{*}{4} & \multirow{9}{*}{$\begin{array}{l}\text { Fisher-filtering } \\
\text { selection }\end{array}$} & \multirow{9}{*}{$\begin{array}{l}\text { thal, number of } \\
\text { major vessels } \\
\text { colored, exercise } \\
\text { induced angina, } \\
\text { max heart rate } \\
\text { achieved, oldpeak, } \\
\text { chest pain type, } \\
\text { slope, sex and age }\end{array}$} & \multirow[t]{9}{*}{9} & \multirow{9}{*}{$\begin{array}{l}\text { TANAGR } \\
\text { A machine } \\
\text { learning } \\
\text { tool and } \\
\text { UCI } \\
\text { Statlog } \\
\text { dataset }\end{array}$} & $B L R-83.33$ & BLR-82.59 \\
\hline & & & & & C4.5-77.41 & C4.5-74.11 \\
\hline & & & & & $C-R T-75.56$ & $C-R T-72.96$ \\
\hline & & & & & SVML- 84.07 & $S V M L-82.59$ \\
\hline & & & & & $S V M P-55.56$ & $S V M P-55.56$ \\
\hline & & & & & SVMR- 82.59 & SVMR - 80.74 \\
\hline & & & & & $S V M S-84.07$ & $S V M S-81.85$ \\
\hline & & & & & $I D 3-70.73$ & ID3 - 70.73 \\
\hline & & & & & $K N N-70.00$ & $K N N-66.30$ \\
\hline
\end{tabular}




\begin{tabular}{|c|c|c|c|c|c|c|}
\hline & & & & & $M L P-82.22$ & $M L P-80.74$ \\
\hline & & & & & $M L R-83.33$ & $M L R-82.59$ \\
\hline & & & & & $N B-84.81$ & $N B-82.59$ \\
\hline & ReliefF selection & sex, thal, resting & 6 & TANAGR & $B L R-83.70$ & $B L R-82.59$ \\
\hline & & electro cardio & & A machine & $C 4.5-82.96$ & $C 4.5-74.11$ \\
\hline & & graphic results, & & learning & $C-R T-79.63$ & $C-R T-72.96$ \\
\hline & & number of major & & tool and & SVML- 84.81 & SVML - 82.59 \\
\hline & & vessels colored, & & UCI & $S V M P-55.56$ & $S V M P-55.56$ \\
\hline & & chest pain type and & & Statlog & $S V M R-80.37$ & SVMR - 80.74 \\
\hline & & exercise induced & & dataset & $S V M S-84.44$ & $S V M S-81.85$ \\
\hline & & & & & ID3 - 70.37 & ID3 - 70.73 \\
\hline & & & & & $K N N-80.00$ & $K N N-66.30$ \\
\hline & & & & & $M L P-83.33$ & $M L P-80.74$ \\
\hline & & & & & $M L R-83.70$ & $M L R-82.59$ \\
\hline & & & & & $N B-83.70$ & $N B-82.59$ \\
\hline 23 & $\begin{array}{l}\text { PSO(Particle } \\
\text { Swarm } \\
\text { Optimization) }\end{array}$ & $\begin{array}{l}\text { Chest pain type, } \\
\text { electrocardiographi } \\
\text { c results, max heart } \\
\text { raterachieved, } \\
\text { exercise induced } \\
\text { angina, oldpeak, } \\
\text { number of major } \\
\text { vessels colored and } \\
\text { thal. }\end{array}$ & 7 & $\begin{array}{l}\text { Weka and } \\
\text { four } \\
\text { different } \\
\text { real } \\
\text { datasets }\end{array}$ & $K N N+P S O-81.4$ & $K N N+\mathrm{PSO}-78.14$ \\
\hline 24 & $\begin{array}{ll}\text { Multi } & \text { Layer } \\
\text { Perceptron } & \end{array}$ & 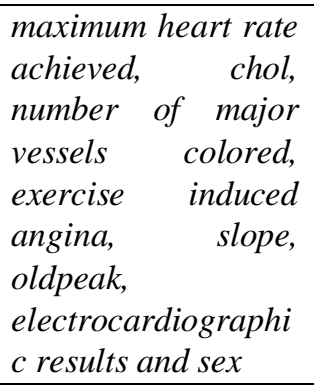 & 8 & $\begin{array}{l}\text { Dataset } \\
\text { from } \\
\text { medical } \\
\text { dataset }\end{array}$ & $M L P-90+$ & $M L P-93+$ \\
\hline 25 & \begin{tabular}{lr}
\multicolumn{2}{l}{ LSTSVM(Least } \\
Square $\quad$ Twin \\
Support $\quad$ Vector \\
Machine)
\end{tabular} & 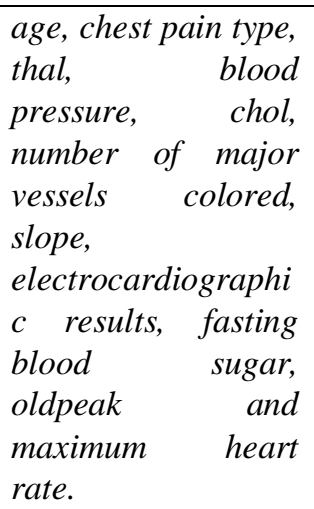 & 11 & $\begin{array}{l}\text { MatlabR20 } \\
12 \mathrm{a} \text { and } \\
\text { Statlog } \\
\text { dataset } \\
\text { from UCI } \\
\text { repository. }\end{array}$ & LSTSVM- 85.18 & $\begin{array}{l}\text { (Please note: Results } \\
\text { are discussed only for } \\
11 \text { attributes) }\end{array}$ \\
\hline
\end{tabular}

\section{PROPOSED APPROACH}

In this work it is proposed to find and recommend a list of relevant attributes for different classifiers which yield high accuracy. Relevant features are determined using the steps given in Fig. 1

(i) Step - 1 Rank the attributes according to correlation measure.

(ii) Step - 2 Perform classification of known data using three commonly used classifiers, namely, MLP, SMO and NB and compare the accuracy of different classifier models.

(iii) Step - 3 Recommend relevant features for the chosen classifiers based on accuracy.

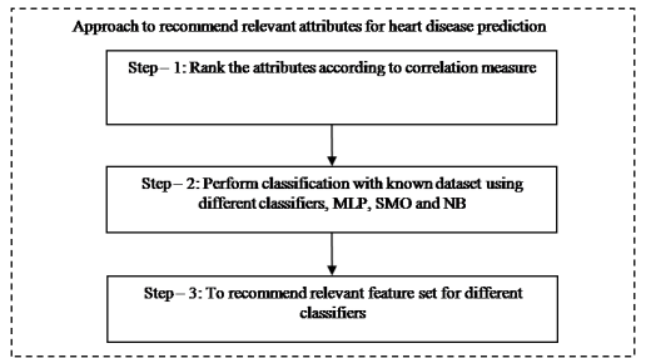

Fig. 1 Proposed Approach 


\section{RECOMMENDATION OF ATTRIBUTES FOR HEART DISEASE PREDICTION USING CORRELATION MEASURE}

To perform the above steps, three experiments have been conducted. It is proposed to use Cleveland dataset and Weka 3.6.9 tool in Windows 7 operating system. Data are collected from Cleveland database of UCI repository [28]. UCI includes four different databases such as Cleveland (303), Hungarian (294), Switzerland (123), and Long Beach VA (200) for heart disease prediction. This database contains 76 attributes. There

class labels are integer, valued from 0 (no presence) to 4(presence). Among these four databases, Cleveland dataset has less number of missing values (only six records contains missing values) than the other datasets. So Cleveland database has been taken up for experiment work. Further the details of attributes of the dataset are given in Table 2.

Table 2 Details of Attributes

\begin{tabular}{|l|l|l|l|}
\hline S.No & Attribute & \multicolumn{1}{|c|}{ Value } & \multicolumn{1}{c|}{ Description } \\
\hline 1 & age & $29-62$ & age in years \\
\hline 2 & sex & 0-male, 1- female & gender \\
\hline 3 & cp & $\begin{array}{l}\text { 1-typical angina; 2-atypical angina } \\
\text { 3-non-anginal pain; 4-asymptomatic }\end{array}$ & chest pain type \\
\hline 4 & trestbps & Numeric value(140mm/Hg) & resting blood pressure in $\mathrm{mm} / \mathrm{Hg}$ \\
\hline 5 & chol & Numeric value(289mg/dl) & serum cholesterol in $\mathrm{mg} / \mathrm{dl}$ \\
\hline 6 & fbs & 1-true, 0-false & \multicolumn{1}{c|}{ fasting blood pressure $>120 \mathrm{mg} / \mathrm{dl}$} \\
\hline 7 & restecg & 0-normal, 1-having ST-T, 2-hypertrophy & resting electrocardiographic results \\
\hline 8 & thalach & 140,173 & maximum heart rate achieved \\
\hline 9 & exang & 1-yes, 0-no & exercise induced angina \\
\hline 10 & oldpeak & Numeric value & $\begin{array}{l}\text { ST depression induced by exercise relative } \\
\text { to rest }\end{array}$ \\
\hline 11 & slope & 1 -upsloping, 2-flat, 3-downsloping & the slope of the peak exercise ST segment \\
\hline 12 & ca & $\begin{array}{l}0-3 \text { vessels } \\
\text { number of major vessels colored by } \\
\text { flourosopy }\end{array}$ \\
\hline 13 & thal & 3-normal, 6-fixed defect, 7-reversable defect & thalassemia \\
\hline 14 & num & $\begin{array}{l}0:<50 \% \text { diameter narrowing } \\
1:>50 \% \text { diameter narrowing }\end{array}$ & $\begin{array}{l}\text { diagnosis of heart disease (angiographic } \\
\text { disease status) }\end{array}$ \\
\hline
\end{tabular}

\section{EXPERIMENTATION AND RESULTS}

There may be many attributes related to a given prediction problem. But not all the attributes have strong association with the prediction. Hence finding the relevant attributes for a given prediction problem is important. In this work, relevant attributes for heart disease prediction are determined using correlation measure. As mentioned above, from literature it is found that the 13 attributes (thal, ca, exang, oldpeak, thalach, $c p$, slope, sex, age, restecg, trestbps, chol, fbs) are being used while predicting heart diseases. In order to find the weight or rank of these attributes an experiment has been conducted. In this experiment the correlation between each attribute and class label is found out. Attributes along with their correlation values are given in Table 3 .

In order to determine which feature set produces optimal accuracy, second experiment is conducted with three popularly used classifiers, namely, NB, MLP and SMO. While doing the above experiment, attributes are added one by one up to 13 attributes by choosing the attribute with highest weight as the first attribute. Accuracy of these classifiers is computed for different feature sets as given in Table 4.

Table 3 Attributes and their weights

\begin{tabular}{|l|l|l|}
\hline S.No & Attribute & Rank \\
\hline 1 & thal & 0.4862 \\
\hline 2 & ca & 0.4608 \\
\hline 3 & exang & 0.4368 \\
\hline
\end{tabular}

\begin{tabular}{|l|l|l|}
4 & oldpeak & 0.4307 \\
\hline 5 & thalach & 0.4217 \\
\hline 6 & cp & 0.3817 \\
\hline 7 & slope & 0.3564 \\
\hline 8 & sex & 0.2809 \\
\hline 9 & age & 0.2254 \\
\hline 10 & restecg & 0.1664 \\
\hline 11 & trestbps & 0.1449 \\
\hline 12 & chol & 0.0852 \\
\hline 13 & fbs & 0.028 \\
\hline
\end{tabular}

Table 4 Accuracy for three different classifiers with different attribute sets

\begin{tabular}{|l|l|l|l|}
\hline \multirow{2}{*}{ Attribute list } & \multicolumn{3}{|l|}{ Classifiers Accuracy in \% } \\
\cline { 2 - 4 } & NB & MLP & SMO \\
\hline thal & 76.5677 & 76.2376 & 76.5677 \\
\hline thal, ca & 79.2079 & 79.538 & 75.5776 \\
\hline thal, ca, exang & 82.5083 & 82.5083 & 78.5479 \\
\hline $\begin{array}{l}\text { thal, ca, exang, } \\
\text { oldpeak }\end{array}$ & 79.868 & 80.8581 & 80.8581 \\
\hline $\begin{array}{l}\text { thal, ca, exang, } \\
\text { oldpeak, thalach, }\end{array}$ & 82.5083 & 79.868 & 84.1584 \\
\hline $\begin{array}{l}\text { thal, ca, exang, } \\
\text { oldpeak, thalach, } c p\end{array}$ & 84.1584 & 79.2079 & 83.8284 \\
\hline
\end{tabular}




\begin{tabular}{|c|c|c|c|}
\hline $\begin{array}{l}\text { thal, ca, exang } \\
\text { oldpeak, thalach, cp, } \\
\text { slope }\end{array}$ & 84.1584 & 81.1881 & 83.8284 \\
\hline $\begin{array}{l}\text { thal, ca, exang } \\
\text { oldpeak, thalach, cp, } \\
\text { slope, sex }\end{array}$ & 84.1584 & 81.5182 & 83.4983 \\
\hline $\begin{array}{l}\text { thal, ca ,exang } \\
\text { oldpeak, thalach, cp, } \\
\text { slope, sex, age }\end{array}$ & 83.4983 & 83.4983 & 83.4983 \\
\hline $\begin{array}{l}\text { thal, ca, exang } \\
\text { oldpeak, thalach, cp, } \\
\text { slope, sex, age, } \\
\text { restecg }\end{array}$ & 84.4884 & 81.8482 & 84.8185 \\
\hline $\begin{array}{l}\text { thal, ca, exang } \\
\text { oldpeak, thalach, cp, } \\
\text { slope, sex, age, } \\
\text { restecg, trestbps }\end{array}$ & 83.8284 & 82.8383 & 84.8185 \\
\hline $\begin{array}{l}\text { thal, ca, exang } \\
\text { oldpeak, thalach, cp, } \\
\text { slope, sex, age, } \\
\text { restecg, trestbps, } \\
\text { chol }\end{array}$ & 83.8284 & 80.5281 & 84.4884 \\
\hline $\begin{array}{l}\text { thal, ca, exang } \\
\text { oldpeak, thalach, cp, } \\
\text { slope, sex, age, } \\
\text { restecg, trestbps, } \\
\text { chol,fbs }\end{array}$ & 83.4983 & 80.8581 & 84.1584 \\
\hline
\end{tabular}

From Table 4, it found that attribute set 9 and attribute set 10 are giving good accuracy. The accuracy values for these attributes sets with NB, MLP and SMO are given in Table 5

Table 5 Accuracy values for different classifiers with attribute set-9 and attribute set-10

\begin{tabular}{|c|c|c|c|c|}
\hline \multirow{2}{*}{$\begin{array}{l}\text { Classifier } \\
\text { Name }\end{array}$} & \multirow{2}{*}{$\begin{array}{l}\text { Selected } \\
\text { attributes }\end{array}$} & \multirow{2}{*}{$\begin{array}{l}\text { Number } \\
\text { of } \\
\text { selected } \\
\text { attributes }\end{array}$} & \multicolumn{2}{|c|}{ Accuracy $\%$} \\
\hline & & & $\begin{array}{l}\text { With } \\
\text { selected } \\
\text { attributes }\end{array}$ & $\begin{array}{l}\text { With } 13 \\
\text { attributes }\end{array}$ \\
\hline NB & $\begin{array}{l}\text { thal, cp, } \\
\text { ca, } \\
\text { oldpeak, } \\
\text { exang, }\end{array}$ & 10 & 84.4884 & 83.4983 \\
\hline
\end{tabular}

\begin{tabular}{|l|l|l|l|l|}
\hline & $\begin{array}{l}\text { thalach, } \\
\text { slope, } \\
\text { age, sex, } \\
\text { restecg }\end{array}$ & & & \\
\hline MLP & $\begin{array}{l}\text { thal, cp, } \\
\text { ca, } \\
\text { oldpeak, } \\
\text { exang, } \\
\text { thalach, } \\
\text { slope, } \\
\text { age, sex }\end{array}$ & & & \\
\hline SMO & $\begin{array}{l}\text { thal cp, } \\
\text { ca, } \\
\text { oldpeak, } \\
\text { exang, } \\
\text { thalach, } \\
\text { slope, } \\
\text { age, sex, } \\
\text { restecg }\end{array}$ & & 83.4983 & 80.8581 \\
& & & \\
\hline
\end{tabular}

\section{INTER-COMPARISON WITH EXISTING METHODS}

Results obtained in this work are inter-compared with feature set represented in literature. From literature, the commonly used feature set are found to be

- Feature set with 6 attributes (thal, cp, ca, oldpeak, exang, thalach)

- Feature set with 7 attributes (thal, cp, ca, oldpeak, exang, thalach, slope)

- Feature set with 9 attributes (thal, $c p, c a$, oldpeak, exang, thalach, slope, age, sex)

- Feature set with 10 attributes (thal, cp, ca, oldpeak, exang, thalach, slope, age, sex, restecg)

- Feature set with 13 attributes (thal, $c p, c a$, oldpeak, exang, thalach, slope, age, sex, restecg, trestbps, chol,

- $f b s)$

In order compare the feature set obtained in the proposed work with that of the above mentioned feature set, third experiment is conducted with above feature set and same classifiers. The accuracy values obtained from the above feature sets for different classifiers are given in Table 6

Table 6 Inter-comparison of the proposed approach with existing methods

\begin{tabular}{|c|c|c|c|c|}
\hline \multirow{2}{*}{ Attribute Name } & \multirow{2}{*}{$\begin{array}{l}\text { No. of } \\
\text { attributes }\end{array}$} & \multicolumn{3}{|c|}{ Classifiers Accuracy in \% } \\
\hline & & NB & MLP & SMO \\
\hline $\begin{array}{l}\text { thal, } c p, c a, \text { oldpeak, } \\
\text { exang, thalach }\end{array}$ & 6 & 84.1584 & 79.2079 & 83.8284 \\
\hline thal, $c p, c a$, oldpeak, exang, thalach, slope & 7 & 84.1584 & 81.1881 & 83.8284 \\
\hline thal, cp, ca, oldpeak, exang, thalach, slope, age, sex & 9 & 83.4983 & 83.4983 & 83.4983 \\
\hline thal, cp, ca, oldpeak, exang,thalach, slope, age, sex, restecg & 10 & 84.4884 & $\mathbf{8 1 . 8 4 8 2}$ & 84.8185 \\
\hline $\begin{array}{l}\text { thal, cp, ca ,oldpeak exang, ,thalach, slope, age, sex, restecg, } \\
\text { trestbps, chol, fbs }\end{array}$ & 13 & 83.4983 & 80.8581 & 84.1584 \\
\hline
\end{tabular}

\section{CONCLUSION AND FUTURE WORK}

In this work, an approach is proposed attributes used for prediction of heart diseases are analyzed using correlation measure. Thirteen attributes for the prediction
Published By: 


\section{RECOMMENDATION OF ATTRIBUTES FOR HEART DISEASE PREDICTION USING CORRELATION MEASURE}

of heart disease are identified from literature. These attributes are ranked according to correlation measure.

Then with different classifiers, accuracy values are obtained for all possible feature sets. It is found that feature set consisting of 10 attributes, thal, $c p, c a$, oldpeak, exang, thalach, slope, age, sex, restecg are recommended as relevant feature set(please refer Table 6feature consisting of 10 attributes are given in bold) for further research. In our further work, it is proposed to use the recommended feature set to study the impact of big data techniques and technologies in enhancing the accuracy of classifiers [29-30].

\section{REFERENCES}

1. V. Manikantan \& S.Latha,"Predicting the Analysis of Heart Disease Symptoms Using Medicinal Data Mining Methods", International Journal on Advanced Computer Theory and Engineering, Volume-2, Issue-2, pp.5-10, 2013.

2. Dr.A.V.Senthil Kumar, "Heart Disease Prediction Using Data Mining preprocessing and Hierarchical Clustering", International Journal of Advanced Trends in Computer Science and Engineering, Volume-4, No.6, pp.07-18, 2015.

3. Uma.K, M.Hanumathappa, "Heart Disease Prediction Using Classification Techniques with Feature Selection Method", Adarsh Journal of Information Technology, Volume-5, Issue-2, pp.22-29, 2016.

4. Hidayet TAKCI, "Improvement of heart attak prediction by the feature selection methods", Turkish Journal of Electrical \& Computer Science, pp.1-10, 2018.

5. Himanshu Sharma, M.A.Rizvi, "Prediction of Heart Disease using Machine Learning Algorithms:A Survey",International Journal on Recent and Innovation Trends in Computing and Communication,Volume5,Issue-8,pp.99-104, 2017.

6. Ms.S.Suguna, Sakthi Sakunthala.N ,S.Sanjana, S.S.Sanjhana, "A Survey on Prediction of Heart Disease using Big data Algorithms", International Journal of Advanced Research in Computer Engineering \& Technology, Volume-6,Issue-3,pp.371-378,2017.

7. R.Sharmila, S.Chellammal, "A Conceptual method to enhance the prediction of heart diseases using big data Techniques", International Journal of Computer Sciences and Engineering, Volume-6, special Issue-4, pp.21-25, 2018.

8. Uma.K, Dr.M.Hanumathappa, "Feature Selection Methods for Heart Disease Prediction with Data mining Techniques", Seventh International Conference on Advanced in Computer Engineering-ACE, 2016.

9. Pinar Yildirim," Filter Based Feature Selection Methods for Prediction of Risks in Hepatitis Disease", International Journal of Machine earning and Computing, Volume. 5, No.4, pp.258-263, 2015. n-to-feature-selection-methods-with-an-example

11. Mark A.Hall, Lloyd A.Smith, "Feature Selection for Machine Learning:Comparing a Correlation-based Filter Approach to the Wrapper". Proceedings of the Twelfth International FLAIRS Conference.

12. Mital Doshi,Dr.Setu K Chaturvedi,"Correlation Based Feature Selection(CFS) Techniques to Predict Student Performance", International Journal of Computer Networks \& Communication,Volume-6,No.3,pp.197-206,2014.

13. Chaitrali S.Dangare, Sulabha S. Apte, "Improved Study of Heart Disease Prediction System using Data Mining Classification Techniques", International Journal of Computer Applications, Volume-47,No.10,pp.44-448,2012.
10. https://www.analyticsvidhya.com/blog/2016/12/introductio

14. Shamsher Bahadur Patel, Pramod Kumar Yadav,Dr.D.P.Shukla, "Predict the Diagnosis of Heart Disease Patients Using Classification Mining Techniques",Journal of Agricultural and Veterinary Science,Volume-4,Issue-2,pp.61-64, 2013.

15. Pediredla Praveen Kumar, Sunita A. Yadwad V V D L Tejaswi, "A Data Mining Technique for Prediction of Heart Disease using Hadoop Mapreduce",International Journal of Computer Application,Volume-6,No.6,pp18,2016 .

16. Aditya Methaila, Prince Kansal, Himanshu Arya, Pankaj Kumar, "Early Heart Disease Prediction using Data Mining Techniques",Computer Science \& Information Technology,pp.53-59,2014.

17. Dr.Durairaj.M, Sivagowry.S, "A Pragmatic Approach of PreProcessing the Dataset for Heart Disease Prediction", International Journal of Innovative Research in Computer and Communication Engineering,Volume-2,Issue11,pp.6457-6465,2014.

18. V.Subha,M.Revathi,D.Murugan, "Comparative Analysis of Support Vector Machine Ensembles for Heart Disease Prediction", International Journal of Computer Science \& Communication Networks, Volume-5(6),pp.386-390.

19. T.Revathi, S.Jeevitha,"Comparative Study on Heart Disease Prediction System using Data Mining Techniques",International Journal of Science and Research,Volume-4,Issue-7,pp.2120-2123,2015.

20. Megha Shahi, Er.Rupinder Kaur Gurm, "Heart Disease Prediction System using Data Mining Techniques- A Review",International Journal of Technology and Computing",Volume-3,Issue-4,pp.73-77,2017.

21. T.John Peter, K.Somusundaram, "Study and Development of Novel Feature Selection Framework for Heart Disease Prediction", International journal of Scientific and Research Publications, Volume-2,Issue-10,pp.1-7, 2012.

22. B.Kavitha, R.Naveen Kumar, "Improving Heart Attack Prediction System using Feature Selection and Data Mining Methods", International Journal of Advanced Research in Computer Science,Volume-1,No.v,pp.455461,2010

23. Divya Tomar and Sonali Agarwal, "Feature Selection based Least Square Twin Support Vector Machine for Diagnosis of Heart Disease", International Journal of BioScience and Bio-Technolgy, Volume-6, No.2, pp.69-82, 2014.

24. Jabbar MA,"Prediction of heart disease using k-nearest neighbour and particle swarm optimization", Biomedical Research,Volume-28,Issue-9,pp.4154-4158.

25. B. Subanya, R R Rajalaxmi, "A Novel Feature Selection Algorithm for Heart Disease Classification", International Journal of Computational Intelligence and Informatics, Volume-4, No.2, pp.117-124, 2014.

26. Kittipol Wisaeng, "Predict the Diagnosis of Heart Disease using Feature Selection and k-Nearest Neighbor Algorithm", Applied Mathematical Science,Volume8,No.83,pp.4103-4113,2014.

27. Lakshmi Devasena,"Performance Evaluation of Memory based Classifiers with Correlation Based Feature Selection Subset Evaluator for smart Heart Disease Prediction", International Journal of Research in Engineering and Technology, Volume-5, Special issue-7, pp.11-17, 2016.

28. http.//archive.ics.uci.edu/ml/datasets/Heart+Disease.

29. Prediction of Diseases using Big Data Analysis

30. Heart Disease Prediction with MapReduce by using Weighted Association Classifier and K-Means. 